\title{
Die Abgeltung der Kaderärzte im Spital
}

\section{Richard O. Binswanger}

Dr. med., Facharzt für Radiologie und Nuklearmedizin, viele Jahre lang Chefarzt für Radiologie, zuletzt während 14 Jahren am Kantonsspital Münsterlingen, Mitglied FMH

Der Autor war während 14 Jahren Chefarzt mit Fixlohn und 14 Jahre mit einem honorarbasierten (gemischten) System, ohne erkennbaren Einfluss auf seine Leistung. Er verhandelte als Präsident des Thurgauischen Vereins der Leitenden Spitalärzte den Kaderarztvertrag de Spital Thurgau AG von 2000-2004.

\section{Korrespondenz:}

Dr. med.

Richard O. Binswanger Radiologie und Nuklearmedizin

Oberer Seeweg 9 CH-8597 Landschlacht

r.binswanger[at]bluewin.ch
Ein heikles und umstrittenes Thema. Voller Emotionen, wie der Autor aus eigener Erfahrung weiss" $[1,2]$. Anhand dreier gängiger Modelle sollen Vor- und Nachteile der Abgeltung beschrieben und eine Wertung vollzogen werden.

\section{Der Fixlohn}

Er wird von den betroffenen Ärzten zumeist vehement abgelehnt. Eine Ursache liegt im Misstrauen gegenüber den Spitalträgern und dem Management. Es wird befürchtet, dass diese die Fixlöhne senken wollen oder müssen. Der Fixlohn soll auch leistungsvermindernd sein. $\mathrm{Zu}$ geringe zahlenmässige Leistung lässt sich allerdings messen. Ihre Korrektur bedingt gute Führung und allenfalls personalrechtliche Massnahmen. In der Mayo Clinic [3] gibt es nur Fixlöhne. Allerdings ist das System marktgerecht. Ärzte, welche auch sonst im Markt hohe Einkommen erzielen, zum Beispiel Augenärzte, erhalten einen höheren Fixlohn als Pädiater. Die Mayo Clinic ist gegenüber den Ärzten aber ein speziell verlässlicher Partner. Der weitaus grösste Gewinn des Fixlohnes ist, dass das ärztliche Entgelt als Treiber für Hast, Schludrigkeit und die Vernachlässigung der Bedürfnisse der Patienten wegfällt. Die intrinsische Motivation der Ärzte kann sich entfalten.

\section{Honorarbasierte Systeme}

Sie überwiegen in der Schweiz. Es gibt sie in reiner Form (nur Honorare), überwiegend aber als Mischform (Fixlohn und Honorare). Hier wird nur die reine Form dargestellt. Sie treibt den Arzt zur (honorarberechtigten) Leistung an. Das ist aus finanzieller Sicht für Spital und Arzt positiv. Es gibt aber auch Nachteile. Es drohen der reduzierte Patientenkontakt, Stress, Überarbeitung und Burnout. Im schlimmsten Fall überträgt sich das auch auf Patienten und Mitarbeiter. Nicht honorarberechtigte Leistungen drohen, vernachlässigt zu werden. Ausserdem kann die extrinsische (finanzielle) Motivation ein Übergewicht erhalten.

\section{Leistungsorientierte Systeme}

Vor bald 20 Jahren wurden leistungsorientierte Abgeltungssysteme vor allem vom Management, den Spitalträgern und einem Teil der politischen Parteien propagiert und in vielen Spitälern eingeführt. Sie finden beim Fixlohn und in honorarbasierten Systemen Anwendung. Sie sollen die Ärzte vermehrt zu wirtschaftlichem Denken veranlassen. Aber: Gerade in der Schweiz gibt es eine ärztliche Tradition der Beschränkung auf das Notwendige. Und «Patients' Needs Come First». Leistungsorientierte Systeme beziehen sich zumeist ausschliesslich auf den finanziellen Erfolg einer Klinik. Sie führen zu einer erheblichen Zusatzbelastung der Kaderärzte. Schlimmer ist aber der mögliche Einfluss auf die Patientenselektion. Und noch schlimmer derjenige auf den ärztlichen Diskurs [4]. Dies hat der Autor miterlebt, obwohl in seinem Spital nur maximal fünf Prozent des Gesamtentgelts vom Bonus-Malus-System betroffen waren!

\section{Wertung}

Aufgrund eigener Erfahrung und theoretischer literaturbasierter Kenntnis ist der Autor heute ein Verfechter des Fixlohnsystems für die Abgeltung der Kaderärzte in Spitälern geworden. Nur zweifelt er an der Verlässlichkeit des Managements und der Kontrollorgane. Schweizer Spitäler sind nicht Mayo. Deshalb sollen auch in Zukunft honorarbasierte Systeme überwiegen. Sie garantieren am sichersten die angemessene Entschädigung der ärztlichen Leistung. Leistungsorientierte Systeme gehören entsorgt, insbesondere auch nach den üblen Erfahrungen in der Wirtschaft, speziell der Finanzwirtschaft.

\section{Literatur}

1 Binswanger RO. Lessons Learned. Teil 1. Schweiz Ärztezeitung 2006;87:12,507-11.

2 Binswanger RO. Lessons Learned. Teil 2. Schweiz Ärztezeitung 2006;87:14, 612-4

3 Berry LL, Selterman KD. Management Lessons from Mayo Clinic. McGraw-Hill; 2008.

4 Faller H. Das Ende der Schweigepflicht. Zeit Magazin 16.5.2012, 12-22. 